\title{
Optimizing Energy Consumption in loT- Based Scalable Wireless Sensor Networks
}

\author{
Vinay Rishiwal, MJP Rohilkhand University, Bareilly, India \\ iD https://orcid.org/0000-0003-2451-4949 \\ Preeti Yadav, MJP Rohilkhand University, Bareilly, India \\ Omkar Singh, Bareilly College, Bareilly, India* \\ B. G. Prasad, B.M.S. College of Engineering, India \\ iD https://orcid.org/0000-0002-6224-5105
}

\begin{abstract}
In the recent era of IoT, energy ingesting by sensor nodes in wireless sensor networks (WSN) is one of the key challenges. It is decisive to diminish energy ingesting due to restricted battery lifespan of sensor nodes. The objective of this research is to develop efficient routing protocol/algorithm in IoT-based scenario to enhance network performance with QoS parameters. Therefore, keeping this objective in mind, a QoS-based optimized energy clustering routing (QOECR) protocol for IoT-based WSN is proposed and evaluated. QOECR discovers optimal path for sink node and provides better selection for sub-sink nodes. Simulation has been done in MATLAB to assess the performance of QOECR with pre-existing routing protocols. Simulation outcomes represent that QOECR reduces E2E delay 30\%-35\%, enhances throughput 25\%-30\%, minimizes energy consumption 35\%-40\%, minimizes packet loss 28\%-32\%, improves PDR, and prolongs network lifetime 32\%-38\% over CBCCP, HCSM, and ZEAL routing protocols.
\end{abstract}

\section{KEYWORDS}

CBCCP, HCSM, IoT, QOECR, WSN, ZEAL

\section{INTRODUCTION}

In upcoming years most of population will reside in town area, this rapid town evolution is now retaining compression on present framework (Sharma et al., 2020). To overwhelm this novel ultimatum, town societies nearby biosphere emphasis on IoT based WSN applications in smart cities to decrease outlays, advance communication, broadcasting and increase services (Singh \& Rishiwal, 2019). There are numerous IoT based applications for smart cities including smart framework, smart transference, smart fitness care and smart power grid (Kizito \& Semwanga, 2020). IoT revolution creates it smoother for town constructions to advance sustainability by redeemable power ingesting (Kumar \& Rishiwal, 2019). Smart power supervision methods custom IoT strategies to attach separate lighting, cooling, fire safety and lighting methods to dominant organize software (Galli, 2020). The 
power organization solicitation acmes fields of large custom and power drifts. Smart construction power organization system can be noteworthy to protect power ingesting. As smarter town structures custom power organization systems, the town will develop more maintainable as entire (Kumar \& Rishiwal, 2019). IoT can improve how town societies consume water. Smart rhythms can advance escape exposure and info truthfulness, evade misplaced income due to extravagance. Auspiciously, IoT is best positioned to create improvements to this field (Bekalli \& Balboul, 2019). Info to be gathered from smartphones, traffic cameras, street sensors to shade transportation happenings in immediate (Singh \& Rishiwal, 2019). Motorists can be distressed of coincidences and focused to streets that are fewer congested (Srinidhi et al., 2019). The possible consequences and assistances of IoT based applications in smart cities are massive, and outcome will be significant. IoT is a system of corporeal substances including devices, buildings, vehicles and other substances (Singh et al., 2019). These substances entrenched by electronics, sensors, software and system connectivity that allows statistics gathering and interchange amid them (Kumar \& Aabanadam, 2019). Data gathering and transmission are accomplished over internet deprived of any assistance of hominids. Beside these outlines, IoT deviates the biosphere from actual extinct substances to intellectual effective substances (Lin et al., 2020). Also, it contributes humanoid the honor of governing each thing about. IoT covers four chief modules sensors, interface, system connectivity and data dispensation (Haseeb et al, 2020). System connectivity is important module of IoT. WSN signifies the main method to accomplish system connectivity in IoT based applications (Panda, 2019). WSN entails of gateways and sensor nodes (SNs). SNs interconnect and provide data to gateway SN. Formerly, gateway gathers data from SNs and conveys to operator by internet (Anjum et al., 2018). The key challenge in WSN is redeemable power ingesting of SNs because of small lifetime of SNs, for that, QoS based Optimization Energy Clustering Routing (QOECR) is proposed to resolve the power ingesting delinquent in WSN (Chinthaluru et al., 2019; Mudasser et al., 2019).

Beneath the contextual of progressively established sensor-based scheme, info dispensation technique, and Internet based scheme, IoT occurred as novel manufacturing model, positively published broadcasting innovation technique for industry and information-based network industry. The aim of IoT is to accomplish info interconnectedness the things in entire world continuously. WSN has intended a communal research due to it integrates conventional info scheme including sensor techniques, embedded scheme, computer-based technology and wireless broadcasting methods. It has features of little cost, no cabling and wide assortment range (Zhang, 2020). IoT has importantly prolonged humanoid cognition and discernment competences for unknown surroundings. It is widely used in numerous arenas including natural disaster early cautionary, ecological monitoring, biomedical, smart home and armed inquiry. In Smart areas Industry, IoTs are positioned in surrounding to pleat information connected to a specified industrial development (ID) termed "Ecosystem". In this process, data collected from IoT is allied with info from another IoTs and other sources. An ID directs a set of dissimilar actions for manifold targets that are connected to each other by superiority instruction associations. Further, an ID requests to schedule occurrence to happen some deadlines (Qawy et al., 2020). Consequently, dissimilar precise time necessities can be allied to IoTs to collect and transmit data, which resemble to a time period, and IoT should be active else in sleep state to reduce energy consumption. This problematic is signified by action scheduling problem for IoTs. During data transmission, the deployed sensors can be static or mobile. In recent eons, IoT technology has been amalgamated a lot using other arenas to enhance communication in terms of resource utilization, network throughput and load distribution. In IoT, numerous physical substances are devoted to renovate info while exploitation Internet (Moutaib et al., 2020). Furthermore, WSN technology offers the groundwork for IoT schemes and ropes in detecting and furtherance the surroundings for corporeal atmosphere. A unified IoT scheme includes sensor nodes (SNs) that agonize disadvantages including imperfect lifetime, area exposure, lesser size and restricted dispensation power. Every SN is premeditated with sensing and data dispensation components for broadcasting with its adjacent nodes over devoted stations (Tekin et al., 2020). SN transmits data either straight or through relays to base 
station (BS), which is advanced to end user via gateway. Henceforth, these wireless networks class stances the issues of central information advancing, organization, control of sink node's deployment and covered area of access points in IoT province (Anjum et al., 2019). Furthermore, energy ingesting in WSN is contrariwise proportional to mesh structure of the nodes. The property of mesh structure by SNs can play a vigorous role to advance lifetime and network sustainability (Galli, 2019). Research in IoT has competence to summarize the identification, detecting technique, artificial intelligence and securely unified systems. In current epoch of IoT, energy consumption by sensor nodes in WSN is one of the main challenges. It is conclusive to reduce energy consumption due to delimited battery lifetime of sensor nodes (Amos et al., 2018).

IoT has been imagined as broadcasting and incorporation of shrewd substances. The supremacy of IoT primes to a new context of imminent amenities and applications (Singh \& Rishiwal, 2019). Numerous substances such as portable headsets, devices, RFID tags are IoT components, which are related to Internet thru wired and wireless networks. To placate the numerous necessities of users, shrewd substances can intellect, pleat and communicate data. Broadcasting relates between substances themselves and people. To achieve intuition into numerous issues associated to tangible world corporeal progressions, IoT has been grasped as a vivacious solution. The scientific growths in IoT field have offered numerous execution contests. Identified information can be transmitted in enquired form or in unceasing mode. This needs vigor proficient broadcasting amid sensors (Lin et al. 2020). Further substances are arrayed in IoT, that's why big volumes of energy are expended in entire process. Therefore, green networking theatres a vital role in IoT to decrease energy ingesting and operative costs, diminish contamination and emanations and make maximum of investigation and surroundings preservation. The comprehension of cost drops to attain networking in terms of energy on scalable network is the objective of research objective. Numerous power proficient methods for WSN have been projected in the current ancient including hierarchy, ad-hoc and exact ones, but these agendas have not observed the prearrangement of substances in reflection of a power effectual IoT (Haseeb et al., 2020).

IoT is observed as a developing technology pointing to link abundant sensor empowered devices and are very significant to deliberate the problems and contests in execution of IoT built networks in shrewd towns. The current centuries have perceived sensors with enlarged stylishness and the capability to pleat information deprived of humanoid interference (Panda, 2019). WSNs are predictable important enablers for IoT pattern since its commencement and has developed a platform for positioning numerous types of significant smart city projects, includes conveyance and wayside applications, intensive care of air contamination, smart space, automatic control schemes, and additional IoT built projects. WSN is utmost recognized methods used inside an IoT scheme which permits a bulky gathering of sensors to separately collect information and obviously way the data to Base station (BS). IoT sensors join to develop WSN supported IoT system and more execute information detecting in its goal region to advance identified data to base station (Chithaluru et al., 2019). Such a information identifying and directing procedure needs a significant exchange of data amid the sensor nodes, subsequent in advanced reduction of power. Therefore, capable power consumption methods are abundant desired to contrivance the idea of IoT. WSN is self-assured and establish dismissed number of multi-functional sensors with imperfect battery and arbitrarily dispersed over a inaccessible area. WSNs consumption can be perceived in consumer applications such as, distant controls, homebased mechanization, computer peripheral and much more. The new sensor assisted schemes emphasis on least energy WSNs and poignant for advancing trade intensive care and diagnostics. Such minimum powered sensors can execute wireless broadcasting and can quickly form a system. Though the novelty for large-volume small powered shrewd projects didn't occur in twentieth period, both academic communal and commercial professed the potential for such networks and mounted cooperative activities to tenacity the edifice problems for projects related to smart networks (Zhang, 2020). 
In today's era IoT is playing a vigorous protagonist, in big gage applications including shrewd workshops, smart metropolises, smart circles, smart networks and much more. In Overall, The Process of IoT is built on supportive roles of many sensors, moveable devices, audiovisual cameras in multiethnic metropolitan for information distribution via internet network (Anjum et al., 2019). In IoT aided system, WSN is measured as the important constituent of IoT prototypical and it have manifold sensors in nifty metropolitan for information organization. Moreover, every sensor node in WSN is particularly used for specific purpose and every sensor node is functioned by battery-operated which primes to power utilization in all smart metropolitan agenda broadcasting for information dispensation. Today's, the structure of mutually digitalization of Homes and Smart Metropolises requires a widespread data gathering for specialist care and rheostat. Earlier for smart metropolitan surveillance, WSN is being used widely in smart metropolises, healthcare, home mechanization, and ecological monitoring. Current review demonstrates that implication of IoT is implemented to develop smart structures with legacy property to advance its sustainability and preserve power (Amos et al., 2018). Smart metropolitan power organization framework customs IoT strategies for conniving unusual heating, refrigeration, connect dissimilar, illumination and fire-safety scheme to pivotal management system to advance the canons. For an illustration, IoT can transmute the way of overwhelming aquatic in town areas by retaining shrewd meters, which are proficient of increasing leak detection and data veracity. Additionally, it provides support to increase the manufacture of numerous corporations and assistances to monitor the refraction of revenue fatalities due to inefficiency in time management for dispensation data (Allam et al., 2020).

\section{Related Work}

(Kakhandki et al., 2019) proposed selection model which diminishes power ingesting per bit and exploits network lifetime, it induces power computation overhead on supportive devices. Adaptive priority-based hop election is implemented prolonging network lifetime. Proposed model signifies similar transceiver optimum scheme that exploits least residual power. Optimum transceiver is implemented in proposed model as:

$$
\begin{aligned}
& \max _{P_{m}^{t}, P_{m}^{n}} n_{a}\left(P_{m}^{t}, P_{m}^{n}\right)=\min P_{\text {sensder }}- \\
& C_{x} P_{m^{t}}, P_{a}-C_{x} P_{m^{n}}
\end{aligned}
$$

Notations and symbols used in Eq. (1) have summarized by author in (Kakhandki et al., 2019).

Energy Efficient QoS based IoT environment (TQEE) has been developed by (Kumar et al., 2017). It achieves long term power utilization beneath suitable QoS restraints in varied applications including environmental monitoring and much more. In this method, first a new method of QoS cognizant sensor job relevancy is presented taking accounts the sensing competences of sensors. Formerly, after suggesting the method of critical attention fixed a job to elect sensors, vigorous conclusions for power utilization optimization are completed immediately. For developing this technique, a relevancy function is used.

$$
r\left(c^{n}, q^{n}\right)=1\left\{\operatorname{dis}\left(X^{n}, Y^{m}\right) \leq r^{n}\right\} \cdot \min \left\{\frac{2 \beta^{m}}{Q\left(\frac{\partial^{m}}{\sqrt{y^{n}}}\right)}\right\}
$$

Symbols and notations used in Eq.(2) have been described by author in (Kumar et al., 2017). 
(Rani et al., 2020) proposed energy efficient scheme ME-CBCCP which customs cluster-based structure and a innovative broadcast algorithm for optimization of power restrictions. We demonstrate that this outline is more flexible and effectual associated to customary chemes for WSNs and applied easily in power proficient IoT. ME-CBCCP minimizes broadcast distance mid all the substances to recompense the energy ingesting in inferior layers of IoT structure. A specific function is used for power ingesting is as:

$$
E_{u}=\sum_{v \in R} r_{u v} \cdot S_{u v} \cdot\left(E_{\text {elec }}^{N N}+\epsilon_{1} \cdot d_{u, v}^{2}\right) \forall u \in N N
$$

Notations used in Eq. [3] have been specified by author in (Rani et al., 2020).

ETERNAL algorithm developed by (Devi et al., 2017). Which transfer data based on gratified. Interrelated data routing on similar path decreases traffic. Henceforth, latency is condensed, outcome in least traffic and preservation of battery power. The consequence of projected work entitlements slight network delay, and advanced power competence. Constancy is also safeguarded that entitlements the projected approach superior to prevailing approaches. An objective function is used to develop ETERNAL scheme is as follows:

$$
O=\max \left(D_{g}^{\prime}-D_{g}^{\prime \prime}\right)+\alpha\left(\frac{T_{i}^{\prime}-T_{i}^{\prime \prime}}{T_{i}^{\prime}}\right)
$$

Notations and symbols used in Eq. (4) have been used by author in (Devi et al., 2017).

(Yang et al.,2018) developed EOSR routing protocol for routing strategy based on multi-factor. EOSR used trust assessment model, path building and path preservation. The trust assessment is accountable for manipulating trust value factors and node's broadcasting performance. The path building expansively reflects the trust factors of node, residual power and hop count of path to discover a dependable and power composed path. When there is a malevolent node or an inadequate power node in the path, path conservation will inform the originate node to maintain a novel advancing route. Trust accuracy evaluation model is accessed as:

$$
D T_{i j}=E\left(B e t a\left(\alpha_{i j}, \beta_{i j}\right)\right)=\frac{\alpha_{i j}+1}{\alpha_{i j}+q \beta_{i j}+2}
$$

Symbolizations used in Eq.(5) have been discussed by author in (Yang et al.,2018).

AFSA algorithm proposed by (Manshahia, 2018) implementing objective function. It trusts on numerous cramming constraints such as remaining power and throughput. A fitness function is reflected which is built on remaining power and regularized throughput of IoT network. Fitness function optimization is desired to recover concert of IoT and evade cramming. The fitness function is as follows:

$$
F_{j}=\sum_{i=1}^{n}\left(w_{1} * t_{i}+w_{2} * E_{i}\right)
$$

where $E$ represents remaining energy, $w_{1}, w_{2}$ specify weight vectors through throughput $t$. 
(Sadek, 2018) developed Hy-IoT protocol which is exploited in IoT structure are established and lengthily studied to amount their recitals with a collective statement of arbitrarily dispersed nodes. This postulation doesn't offer a fortuitous to professionally exploit the liveliness of nodes expressly in IoT based applications that have certain type of manifold level of power heterogeneity includes inactive RFID, mobile phones and sensors. Consequently, Hy-IoT offers the alteration in electing the steering based on offering two areas having SDN devices to manage energy and other has standard arbitrarily dispersed consistent nodes which comprise customary restricted power nodes and progressive nodes.

I-AREOR protocol is proposed by (Chithaluru et al., 2020) which implements the novel idea of a hierarchical clustering scheme in which the number of clusters operative with forwarder nodes is directly cavernous for data broadcast. Clusters are identified regardless of their erection and measurement of the sensor node positioned. Node having extraordinary power and less distance would be nominated as CHs. For every sensor node $d p_{i}$, dual restrictions are projected, one is area thickness $d m$ and other is distance $\Theta \mathrm{m}$. The area thickness is precisely calculated using two approaches as:

$$
\begin{gathered}
d_{m}=\sum_{n \in I_{n \backslash m}} \mu\left(d_{m n}-d_{c o}\right) \\
f(x)= \begin{cases}1, & d_{m}<0 \\
0, & d_{m}>0\end{cases}
\end{gathered}
$$

The specific symbols and notations used in Eq. (7)-(8) have been summarized by author in (Chithaluru et al., 2020).

(Qureshi et al., 2020) developed GCEEC protocol for farming exactness WSN to recover the weight complementary amid CHs and power ingesting of entire network. GCEEC protocol picks the effectual position of $\mathrm{CH}$ adjacent the power centroid location and for gateway sensor node election for communicating data to BS through multi-hop transmission which exploits the $\mathrm{CH}$ exposure and decreases transmission energy of $\mathrm{CH}$. It is separated into two subdivision network setup components and process component. Setup component grants the power ingesting model, power centroid location, weight gateway node and $\mathrm{CH}$ connection weight used in GCEEC. The processing component elucidates the setup, broadcast and rotation phase. Energy representation in GCEEC is implemented as:

$$
\begin{aligned}
& X_{e c}=\frac{\sum_{i=1}^{n}\left(E_{i_{r s}} / E_{0}\right) X}{N} \\
& Y_{e c}=\frac{\sum_{i=1}^{n}\left(E_{i_{r s}} / E_{0}\right) Y}{N}
\end{aligned}
$$

Symbolizations used in Eq. (9)-(10) have been summarized by author in (Qureshi et al., 2020). WSN-IoT paradigm developed by (Jain et al., 2017) for real time applications. The chief emphasis is to discover for giving material as prompt as possible to destination which is requirement in smart cities. It is based on optimum route which is elected by TOPSIS optimization and attains balance amid recital and power ingesting with respect to four standards: Smallest distance of nominated nodes from 
BS, remaining power, least Euclidean distance amid $m^{\text {th }}$ nominated node and subsequent optimal $n^{\text {th }}$ node in broadcast and adjacent nodes. Decision matrix is used in this protocol is as:

$$
r_{i j}=\frac{x_{i j}}{\sqrt{\sum_{i=1}^{n} x_{i j}^{2}}} \ldots \ldots \ldots \ldots \ldots \ldots
$$

Precise symbols and symbolizations used in Eq. (11) have been specified by author in (Jain et al., 2017).

Chain based Cluster Cooperative Protocol (CBCCP) (Rani et al., 2015) minimizes the energy consumption and prolongs network lifetime. Using unvarying dissemination of power ingesting, CBCCP has improved network lifetime of every node and henceforth it provides network constancy. Sensor nodes apprise their adjacent sets on inter and intra cluster-level. By adjusting the broadcasting at cluster and sub-cluster level nodes can unceasingly transmit data to base station. Clustering is exaggerated in case of $\mathrm{CH}$ drains energy up to the threshold level. It is activated using re-selection $\mathrm{CH}$ algorithm. Novel $\mathrm{CHs}$ are selected in every cluster. Clusters are presumed in exact magnitudes and stationery. Therefore, clustering procedure concludes in single recapitulation, which is dissimilar with leach-based protocols. Optimal path and total consumed energy can be calculated as:

$$
P_{\text {opt }}=\left(\sqrt{A_{l}^{2}+4 A_{b}^{2}} \times \sqrt{L^{2}+B^{2}}\right) / 2 A_{b}
$$

$$
E_{d}=\left(\left(n \times A_{l} \times A_{b}\right) / 2 L B\right) \times \sqrt{L^{2}+B^{2}}
$$

Specific symbols and notations used in Eq. (12)-(13) have bben used by author in (Rani et al., 2015).

Hierarchical Computation Strategic Making (HCSM) (Sudhari \& Jaikumar, 2019) is reflected as a projecting module for increasing IoT idea and the current enhancement in smart workstation systems that renovates extreme calculating power for enactment process and high memory perimeters. Furthermore, the IoT ideas near to cyber security scheme, which objectives reliable integrated physical outlines with dispensation and communication possessions. Moreover, in urban regions for smart city observing, IoT sensor treat as a provision idea and the incorporation of the software demarcated sensor networks using tradition of WSN based outlines is encouragement to modification the normal city facilities tends smart cities. The objective function is used for developing HCSM is as follows:

$$
G=\sum_{j=1}^{b} \sum_{l=1}^{m} \phi_{j l}\left(S_{j, l}^{u}\right)+\delta \sum_{j=1}^{b} \sum_{l=1}^{m} \phi_{j l} \ln \phi_{j l}
$$

Notations used in Eq. (14) have been elaborated by author in (Sudhari \& Jaikumar, 2019).

Zone based Energy Aware data Collection (ZEAL) advances (Allam et al., 2020) data transfer and power utilization. It is used for circumstances of portable nodes that gather data from sensors. ZEAL route is unnatural to a conservative line. ZEAL projected a duty-cycle tool to decrease power utilization. The duty-cycle tool smears sleep/wake-up periods slots on associated nodes, where associated node wakens to transmit data and move to sleep mode till sleeping period turns out. Sleeping duration matches to [(half-cycle time $\times$ current time $) \times 2$ ]. Mobile-sink travels out from extant zone 
to move in subsequent one, it directs POLL2 message to last sub-sink node in present zone. When the previous sub-sink node obtains POLL2, it decreases sleeping period with one another. Formerly, it transmits SLP message covering zone-ID and sleeping period to all associated nodes in identical zone. Every associated node obtains SLP message retransmissions to secondary associated nodes till all associates inside the zone move to sleep. (Yassine et al., 2018) presented an innovative platform to enable pioneering analytics on data gathered from smart home appliances. A smart home is well established place of connected appliances and devices which generates a momentous amount of data. They proposed the usage of integration of nodes to cloud system to allow data driven services and identifies the challenges of complexities and resources demand for online and offline data processing, storage and classification analysis. This paper discusses the necessities and design apparatus of the system. They acquired the data from a real smart home of the Vancouver, Canada as case study to present the meaningful results.

IoT applications that are not able to meet the requirements of today's era are being emerged with IoT based computing techniques. This amalgamation needs analysis of various solutions of the difficulties and challenges. A combined architectural model based on a new organization was presented by (Bellavista et al., 2018). This organization compares a huge number of solutions for the development of IoT applications. (Farahani et al., 2018) presented the usability of IoT in healthcare. They proposed an IoT eHealth ecosystem architecture. Inadequate and less effectual services of healthcare are not sufficient to cure the chronic diseases of growing population which is making healthcare the most challenging application of IoT. This article suggests a patient centric treatment instead of clinic centric treatment in which the patients, hospitals and services are treated as objects and they are connected with each other through internet. This patient's centric treatment systems connect enormous entities together resulting as vast amount of data. For the organization and processing of that data there is proposed a multi-layer architecture which includes devices, and cloud computing. This integration reduces the problem of congestion and increased latency.

To overcome the problem of traffic congestion and increased latency in IoT applications are introduced. In such systems nodes are deployed at the edge of the network. Due to the deployment at dispersed unsecure location these nodes are easy target for attackers, (Viejo \& Sanchez, 2019) proposed the concept of orchestration. Orchestration enables the network to be self-tailored for the delivered services to achieve a safe and efficient delivery of services. The article illustrates a number of safe and protected protocols to IoT services through orchestration. The mainly exigent scenario of exchanging the data openly in potentially unsecured networks which can be easily targeted by attacker are considered for the assumption. (Oma et al., 2018) proposed TBFC model. With the help of this tree-based computing model the distribution of the processes and data collected from sensors can be efficiently managed. This specified model reduces the electric energy consumption while delivering the data to the servers and nodes. The analysis fallouts that the consumption of energy is less in the TBFC model in comparison with cloud model. (Kumari et al., 2018) categorized the healthcare industry between 1.0 to 4.0 generations. Healthcare 3.0 has been considered as hospital centric which requires multiple visits of the patients to the hospitals which affect the patients in physical as well as economic aspects. With the technological evolution of IoT, it has been almost painless to implement patient centric treatment. The article provides an analysis regarding the migrated functioning of computing on IoT application to facilitate uninterrupted services to the end users on their ease. The authors proposed a three-layer architecture of patient driven healthcare for real time collection, processing and collection of data.

IoT based routing techniques with its merits and demerits have been given in Table 1 .

\section{PROPOSED ROUTING PROTOCOL (QOECR)}

QOECR performs by saving energy and wake up time by implementing two techniques: minimizing setup phase time and mechanism based on duty cycle. QOECR handle sink layout path and give the 
Table 1. loT based evaluation of diverse routing Techniques

\begin{tabular}{|c|c|c|}
\hline Approach & Objective & Drawback \\
\hline $\begin{array}{l}\text { Selection Model (Gali, } \\
\text { 2019) }\end{array}$ & $\begin{array}{l}\text { Improves network lifetime, decreases overhead } \\
\text { and delay rate. }\end{array}$ & $\begin{array}{l}\text { Model is restricted only for homogeneous } \\
\text { scenario. }\end{array}$ \\
\hline $\begin{array}{l}\text { TQEE (Kumar et al., } \\
\text { 2017) }\end{array}$ & $\begin{array}{l}\text { Reduces energy consumption, prolongs } \\
\text { network lifetime. }\end{array}$ & $\begin{array}{l}\text { Network size is limited and should be } \\
\text { checked on large size of network. }\end{array}$ \\
\hline $\begin{array}{l}\text { ME-CBCCP (Rani et } \\
\text { al., 2020) }\end{array}$ & $\begin{array}{l}\text { Prolongs network lifetime, minimizes delay } \\
\text { and improves PDR. }\end{array}$ & Protocol is not scalable. \\
\hline $\begin{array}{l}\text { ETERNAL (Devi et al., } \\
\text { 2017) }\end{array}$ & $\begin{array}{l}\text { Minimizes delay and energy consumption, } \\
\text { enhances network lifetime, reduces network } \\
\text { lifetime. }\end{array}$ & Performance degrades in dynamic network. \\
\hline $\begin{array}{l}\text { EOSR (Yang et al., } \\
\text { 2018) }\end{array}$ & $\begin{array}{l}\text { Improves PDR, power utilization and better } \\
\text { throughput. }\end{array}$ & $\begin{array}{l}\text { On increasing network size, network } \\
\text { performance degrades. }\end{array}$ \\
\hline $\begin{array}{l}\text { AFSA (Manshahia, } \\
\text { 2018) }\end{array}$ & $\begin{array}{l}\text { Increases network lifetime, throughput and } \\
\text { network reliability. }\end{array}$ & $\begin{array}{l}\text { Performance degrades due to malicious } \\
\text { nodes. }\end{array}$ \\
\hline Hy-IoT (Sadek, 2018) & $\begin{array}{l}\text { Enhances network lifetime and throughput, } \\
\text { minimizes delay. }\end{array}$ & $\begin{array}{l}\text { Protocol is restricted only for } \\
\text { heterogeneous network. }\end{array}$ \\
\hline $\begin{array}{l}\text { I-AREOR (Chithalru, } \\
\text { 2020) }\end{array}$ & $\begin{array}{l}\text { Maximizes network lifetime, balances power } \\
\text { ingesting, and reduces delay. }\end{array}$ & $\begin{array}{l}\text { Network performance compromises on } \mathrm{CH} \\
\text { changing state. }\end{array}$ \\
\hline $\begin{array}{l}\text { GCEEC (Qureshi et al., } \\
\text { 2020) }\end{array}$ & $\begin{array}{l}\text { Reduces traffic load, gives better performance } \\
\text { in agriculture field, improves throughput, } \\
\text { energy ingesting. }\end{array}$ & It works on limited size of network. \\
\hline $\begin{array}{l}\text { WSN-IoT (Jain et al., } \\
\text { 2017) }\end{array}$ & $\begin{array}{l}\text { Optimizes data route, reduces delay, improves } \\
\text { network reliability }\end{array}$ & Unable to support scalability \\
\hline $\begin{array}{l}\text { CBCCP (Rani et al., } \\
\text { 2015) }\end{array}$ & $\begin{array}{l}\text { Reduces energy consumption, improves } \\
\text { network lifetime and network reliability. }\end{array}$ & $\begin{array}{l}\text { Need to check performance in } \\
\text { heterogeneity scenario }\end{array}$ \\
\hline $\begin{array}{l}\text { HCSM (Sudhari et al., } \\
\text { 2019) }\end{array}$ & $\begin{array}{l}\text { Enhances network lifetime, balances network } \\
\text { load, supports scalability }\end{array}$ & $\begin{array}{l}\text { Need to enhance security for the reliability } \\
\text { of network. }\end{array}$ \\
\hline $\begin{array}{l}\text { ZEAL (Allam et al., } \\
\text { 2020) }\end{array}$ & $\begin{array}{l}\text { Improves network lifetime, minimizes delay, } \\
\text { enhances throughput. }\end{array}$ & $\begin{array}{l}\text { Degrades network performance on large } \\
\text { size of network. }\end{array}$ \\
\hline QOECR & $\begin{array}{l}\text { Reduces E2E delay, enhances throughput, } \\
\text { minimizes energy consumption, minimizes } \\
\text { packet loss, improves PDR and prolongs } \\
\text { network lifetime. }\end{array}$ & - \\
\hline
\end{tabular}

best performance in terms of power utilization and data delivery. It finds the optimal path to maintain balance amid all parameters. QOECR includes three main phases: pre-processing, setup and data aggregation phase are discussed below:

\section{Pre-Processing Phase}

In this phase QOECR searches the optimum routes for sink node, optimum route is demarcated which attains least number of hops and least distance amid sink and sensor nodes. Firstly, distribution of sink node and member nodes is obtained and get optimum P paths. Centers of locations and clusters are identified, post connected centers, estimated angles and set the route. It searches fixed amount of clusters $\mathrm{C}$ in the existing data set. A particular cluster is demarcated aggregation of data points convened by definite similarities. $\mathrm{C}$ denotes number of essential centroids in dataset that is midpoint of cluster. In IoT based WSN, sink nodes and member nodes location signifies dataset. Centroids are fixed positions having least hop and distance amid sink and sensor nodes. 


\section{Setup Phase}

QOECR uses priority equation as given in Eq. (4) for sink node election. Priority depends on per sub-sink node members $(\boldsymbol{M})$, number of hops amid member and sub-sink nodes $(\boldsymbol{N})$ and $\boldsymbol{\beta}$. Weight parameter is used to maintain balance amid $\boldsymbol{M}$ and $\boldsymbol{N}$. Though, QOECR unable to decide value of $\boldsymbol{\beta}$, therefore we find optimum value for $\boldsymbol{\beta}$. It has been seen for large network $\boldsymbol{\beta}$ values vicissitudes. Consequently, it is needed to amend and implementing priority normalization on $\boldsymbol{M}$ and $\boldsymbol{N}$. Both parameters hold similar weights in the sink node election. Priority is unswervingly related to $\boldsymbol{M}$ and reversely to $N$. Therefore, priority normalization is performed as:

$$
N_{r}=\frac{M}{\max (M)}+\frac{1 / N}{1 / \min (N)}
$$

Modify sub-sink priority election adds distance amid member nodes and sub-sink. It should not be dependent only on $\boldsymbol{N}$ due to member nodes may elect sub-sink having least number of hops but at lengthier distance which consumes more energy. Therefore, distance $\boldsymbol{D}$ is again critical parameter in the election of sub-sink as well $\boldsymbol{M}$ and $\boldsymbol{N}$. Distance amid member and sub-sink nodes can be calculated as:

$$
D\left(d_{1}, d_{2}\right)=\sqrt{\left(x_{2}-x_{1}\right)^{2}+\left(y_{2}-y_{1}\right)^{2}}
$$

where $(x 1, y 1)$ denotes location of member node $d 1$ and $(x 2, y 2)$ specify location of sub-sink node $d 2$.

Alike hops, priority is reversely relative to distance as power utilization enhances to cover a lengthy distance. Therefore, normalized distance can be calculated as:

$$
N_{r}=\frac{M}{\max (M)}+\frac{1 / N}{1 / \min (N)}+\frac{1 / D}{1 / \min (D)}
$$

\section{Data Aggregation phase}

After pre-processing phase data aggregation phase is performed, where sub-sink nodes gather data and transmit to sink node in a definite time period. Every member estimate priority $N_{r}$ of all sub sink nodes inside the same zone using Eq. (18). Every member node elects sub-sink node using highest priority. Sub-sink node transmits data packets until allocated time period is terminated or data packet queue is exhausted.

$$
N_{r}=\beta \times M+\frac{(1-\beta)}{N}
$$

\section{SIMULATION FRAMEWORK}

To examine effectiveness of proposed routing protocol an applicable configuration is needed to spectacle picturing of outcomes as given in Fig 1 . 
Figure 1.

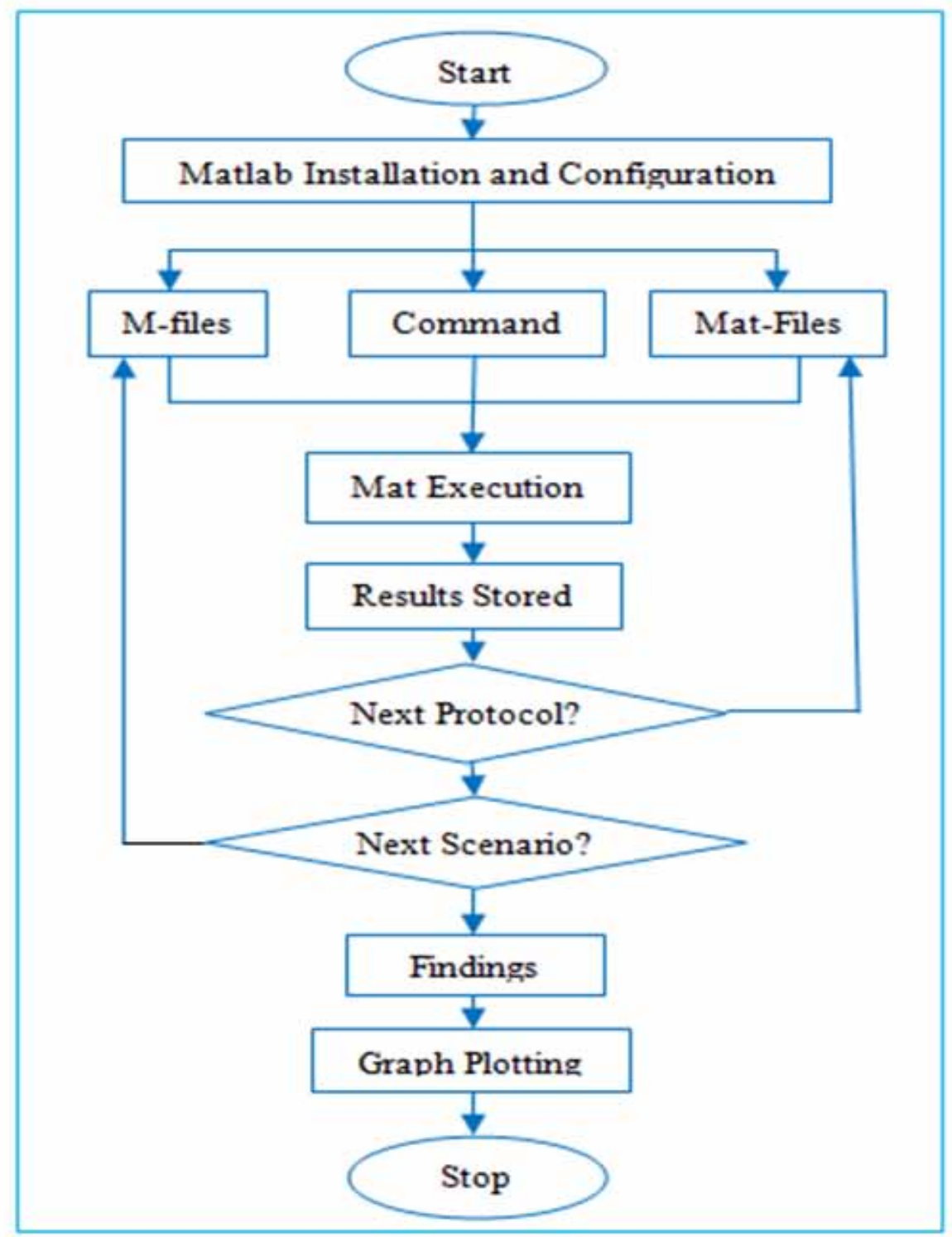

The simulation has been done in MATLAB version 8.1 (https://in.mathworks.com/discovery/ modeling-and-simulation.html) for numerous network size 100-1000. Sensors are deployed in sensing field $200 * 200 \mathrm{~m}^{2}$. Dissimilar constraints for assessing the efficiency of QOECR are shown in Table 2

\section{PERFORMANCE METRICS \& RESULT DISCUSSION}

Concert metrics used to discover the outcome over dissimilar constraints for QOECR are quantified as follows: 
Table 2. Simulation Parameters

\begin{tabular}{|l|l|}
\hline \multicolumn{1}{|c|}{ Parameters } & \multicolumn{1}{c|}{ Values } \\
\hline Simulation Area & $200 * 200 \mathrm{~m}^{2}$ \\
\hline Number of Nodes & $100-1000$ \\
\hline Coverage Area & $200 \mathrm{~m}$ \\
\hline Channel Type & Wireless \\
\hline Distribution of Node & Random \\
\hline Initial Energy & 1 Joules \\
\hline Packet Size (Byte) & 200 \\
\hline Communication Channel & Bi-directional \\
\hline Type of Antenna & Omni directional antenna \\
\hline Radio Broadcast Model & Two-way ground \\
\hline Routing Protocols & CBCCP, HCSM, ZEAL and QOECR \\
\hline
\end{tabular}

\section{Number of Nodes}

offers network size; sensors can be improved inside the sensing area deprived of debasing the network performance.

\section{End-to-End Delay}

It bounces the disruption of packet endorsing queue amid sender to receiver and least packet delay decreases power exploitation.

\section{Throughput}

Data packets volume transmitted positively from source to destination in a definite period.

\section{Energy Consumption}

Overall power depletion by network for different nodes can be premeditated as:

$$
\mathrm{A}_{\mathrm{Tx}}(\delta, \mathrm{L})=\left\{\begin{array}{l}
\delta \hat{\mathrm{A}}_{\text {elec }}+\delta \mu_{f s} \mathrm{~L}^{2}, L \leq \mathrm{L}_{0} \\
\delta \dot{\mathrm{A}}_{\text {elec }}+\delta \mu_{m p} \mathrm{~L}^{4}, L>\mathrm{L}_{0}
\end{array}\right.
$$

$$
\mathrm{A}_{\mathrm{Rx}}(\delta)=\delta \hat{\mathrm{A}}_{\text {elec }}
$$

$\mathrm{A}_{\text {elec }}$ demonstrate power ingesting communicating data to sink node and $\mu_{f s}$ signifies coefficient amplification of free space signal, $\mu_{m p}$ spectacles multipath declining coefficient and values are customary rendering to circuit amplifier. $L$ indicates space amid transmitter and receiver and $\delta$ illustrates poignant data in bit form.

\section{Packet Loss}

It delivers packets stirring transversely a system and failures to reach their endpoint. 


\section{Packet Delivery Ratio}

$P D R$ is a significant aspect to squared distinction of QOECR. If entire amount of received packets and sent packets be $P_{R}$ and $P_{S}$ correspondingly, then $P D R$ can be premeditated as:

$$
P D R=\frac{P_{R}}{P_{S}}
$$

\section{Network Lifetime}

Lifetime of network can be represented in the form of exhausted nodes, during the simulation minimum number of exhausted nodes enhances network lifetime.

According to simulation outcomes distinctive courtesy has been specified on real time concert of data transmitting with minimum energy consumption. In proposed protocol QOECR end-to-end delay is preserved at precise level (less than 1 second). In the specified Fig. 2, E2E delay extends on enhancing number of sensors, but performance of network is not prompted because of dissimilar network size and stabilities lifetime of network. QOECR achieve improved performance and decreases E2E delay then pre-existing routing protocols CBCCP, HCSM and ZEAL. Therefore, data could be transmitted quickly for real time IoT based network. As exposed in Fig. 3 the throughput of QOECR is greater than CBCCP, HCSM and ZEAL. High throughput delivers higher data communication for maintaining network reliability in the field of IoT.

Figure 2.

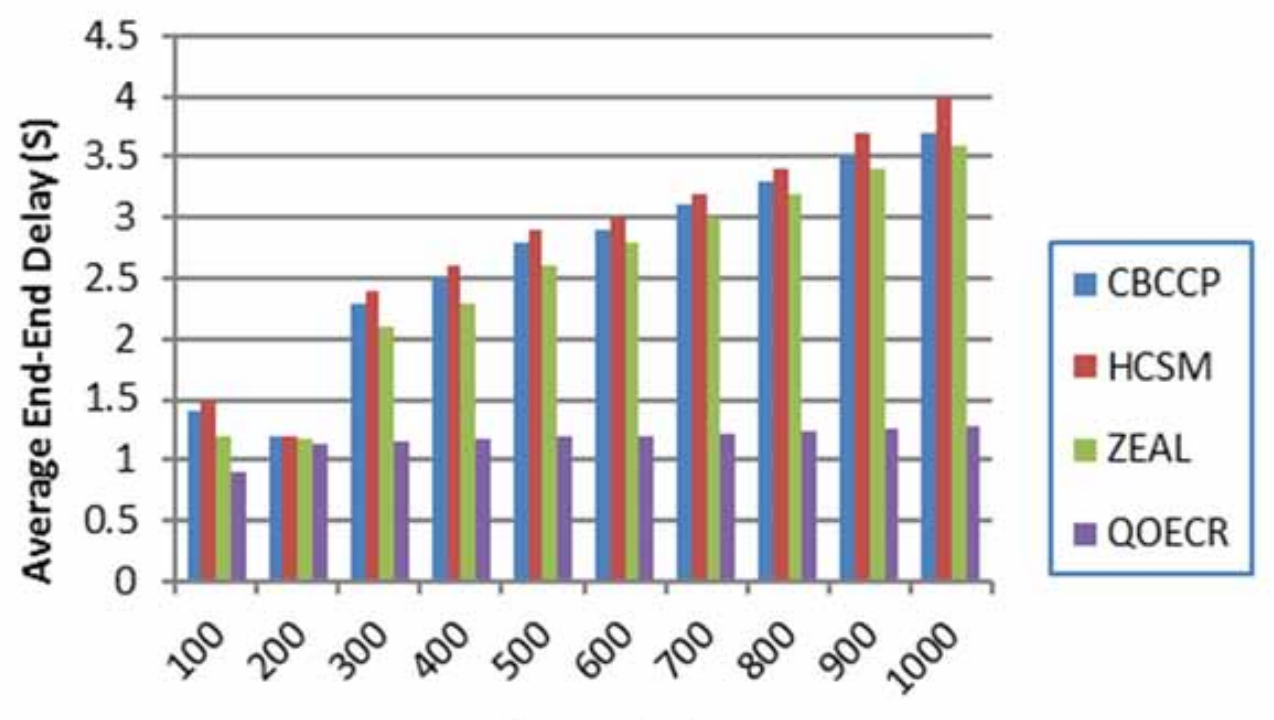

Number of Nodes 
Figure 3.

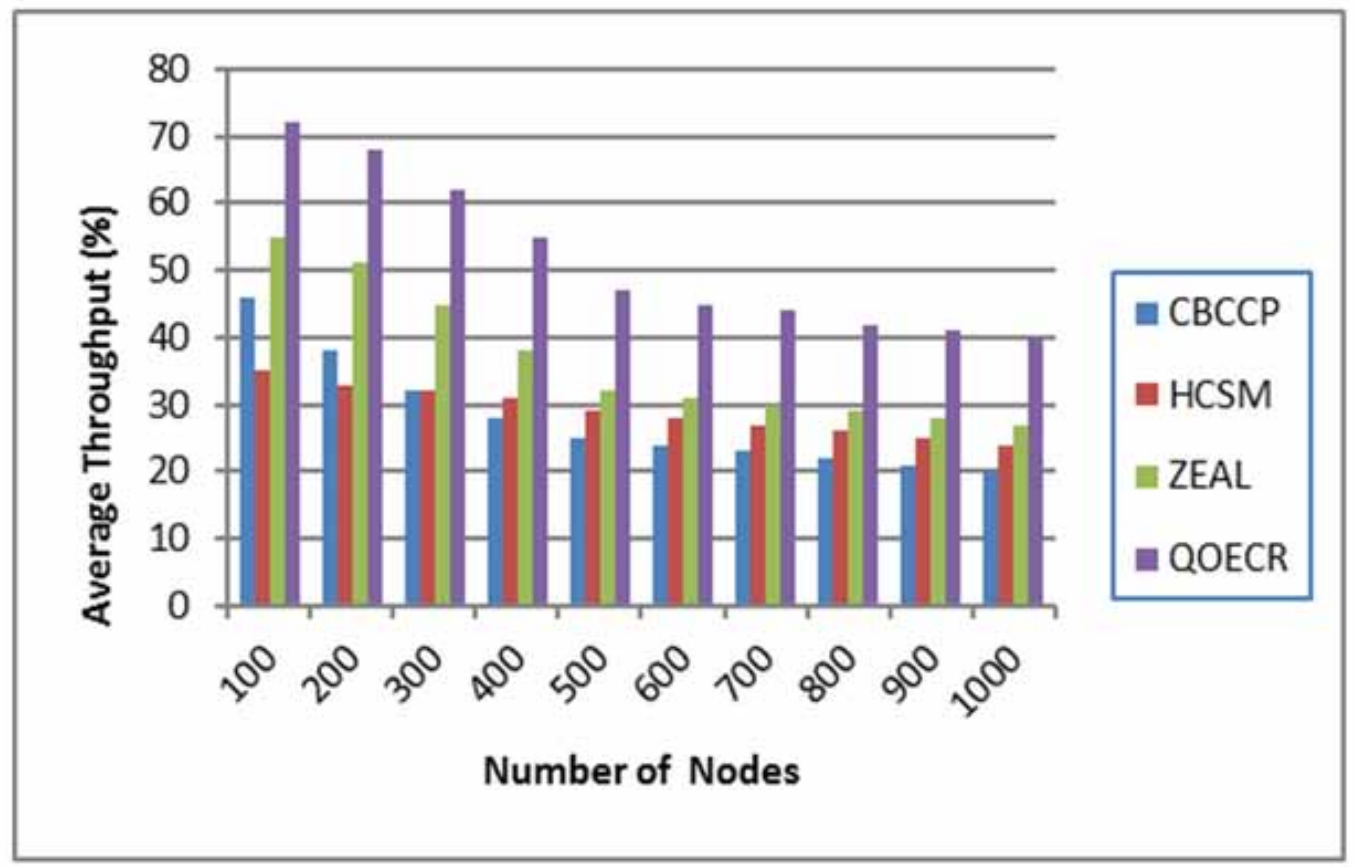

The concert Energy ingesting of simulated protocols CBCCP, HCSM, ZEAL and QOECR is exposed in Fig.4. QOECR provides better performance by balancing energy exploitation in sensing area and protects power so that lifetime of network could be enriched. QOECR diminishes energy consumption and disregards squat energy as subsequent hop node. Power ingesting also improves in higher E2E delay. Consequently, QOECR avoids accelerating data to those regions to stabile power exploitation in entire network.

When network aspects the condition of cramming and consignment of sensor nodes, there is extremely fortuitous of packet damage and jumbles packets. QOECR overlooks unacceptable routes for packets transmitting and equilibriums capacity of nodes to evade cramming. Consequently, the proposed routing protocol QOECR offers improved outcomes as exposed in Fig.5 and decreases packets forfeiture than CBCP, HCSM and ZEAL, so that indispensable finding info could be transmitted in IoT based scenarios.

Fig. 6 signifies packet delivery ratio on dissimilar size of networks. PDR is unrelenting in QOECR than CBCCP, HCSM and ZEAL routing protocols and offers constancy of data to entire network. QOECR communicates enormous numbers of data packets with squat power ingesting which delivers effectiveness of network.

Further, network lifetime is an important parameter inside the network. Essential information can be aggregated during activated network. Once, network fails then sometime critical information losses. Fig. 7 represents network lifetime in the form of number exhausted nodes. If number of exhausted nodes increases in the network that means network performance is degrading and affects network reliability. QOECR provides better performance in network lifetime than pre-existing protocols CBCCP, HCSM and ZEAL. 
Figure 4.

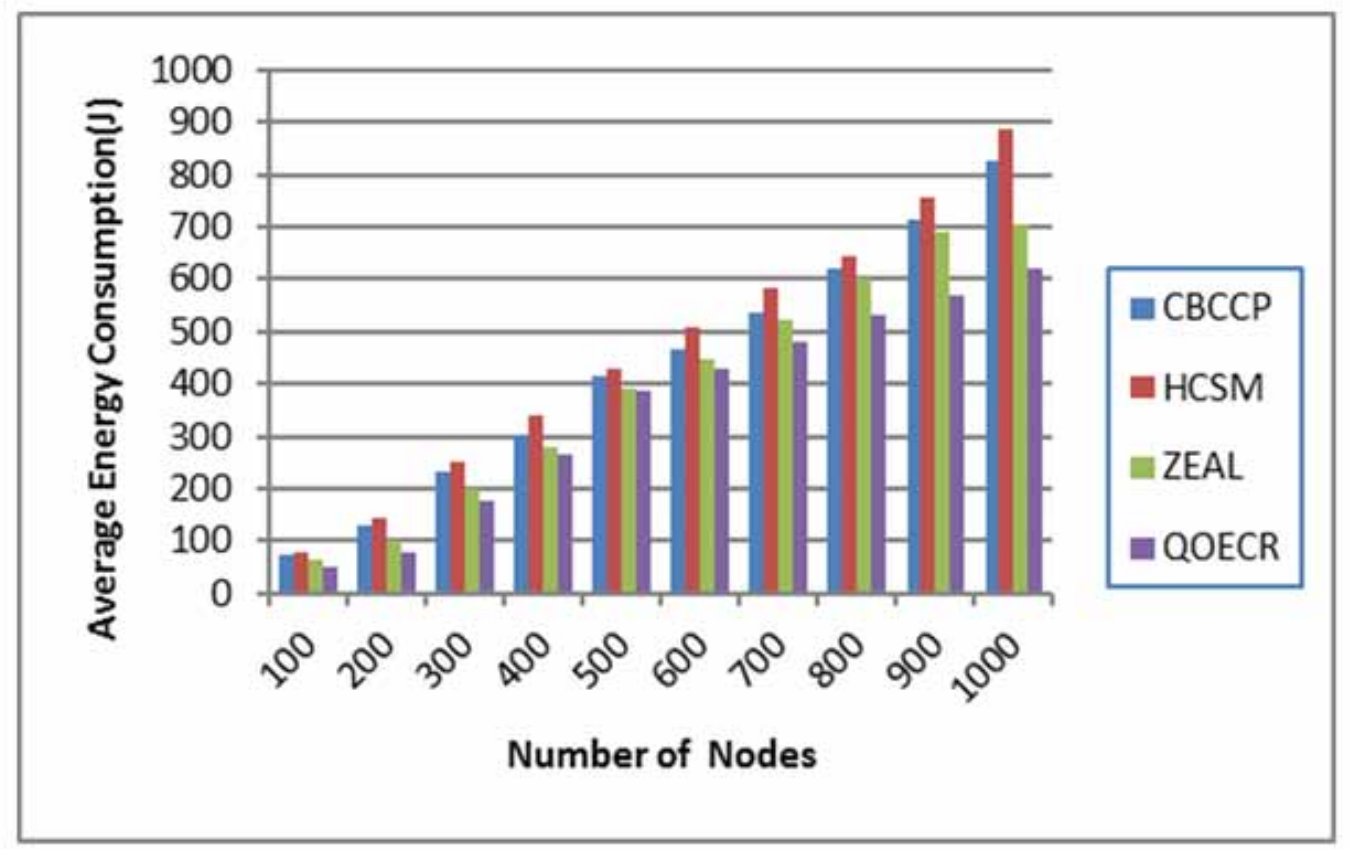

Figure 5.

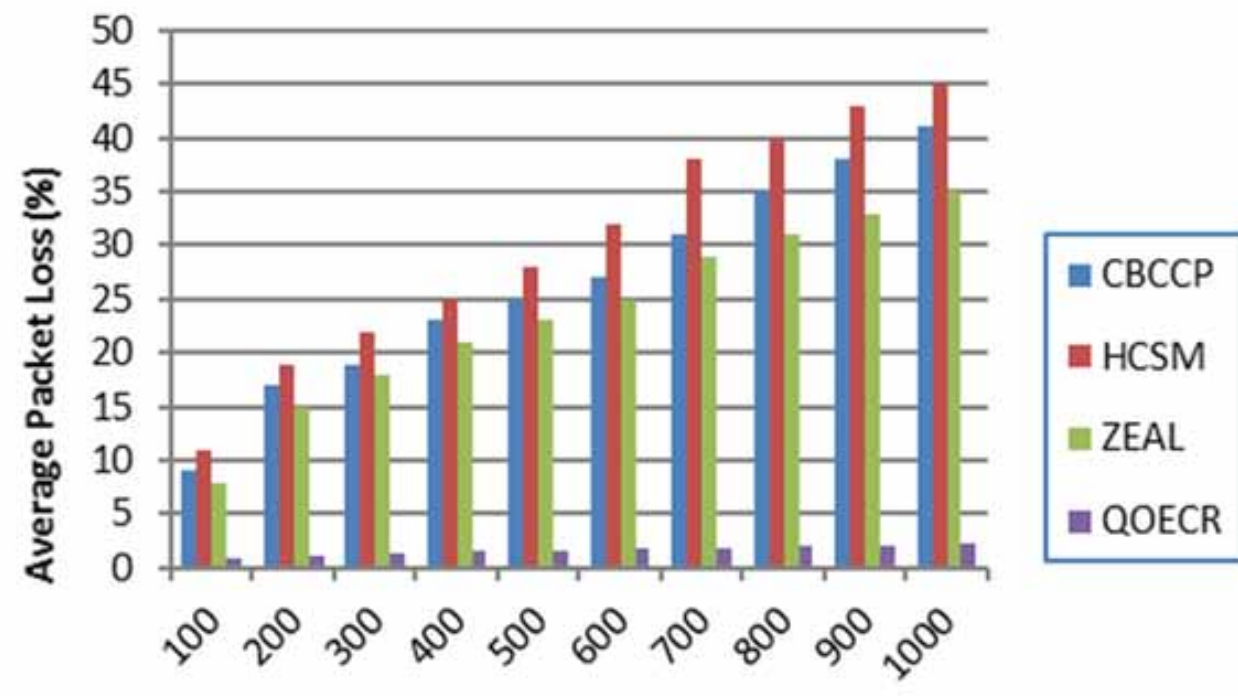

Number of Nodes 
Figure 6.

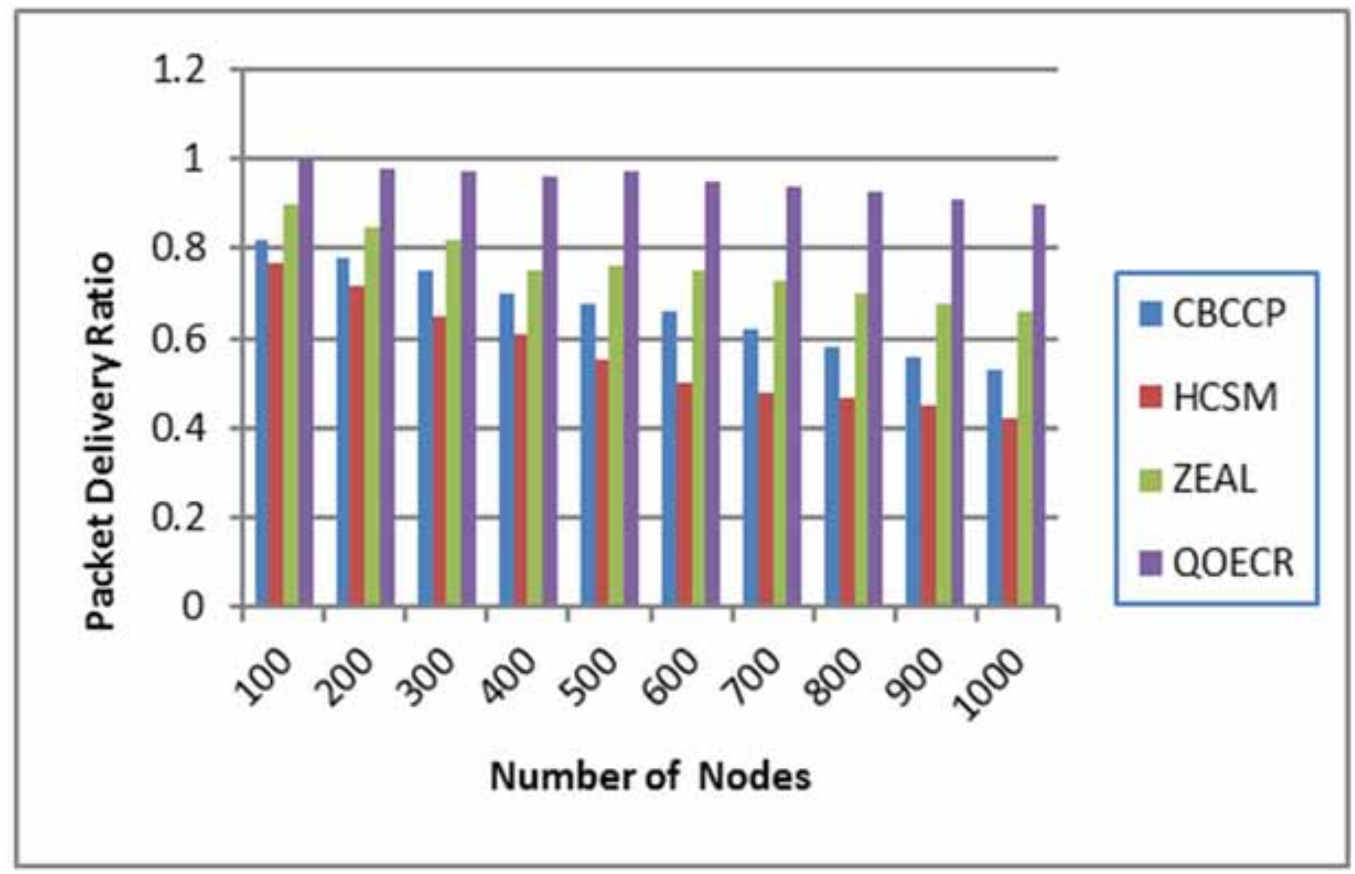

Figure 7.

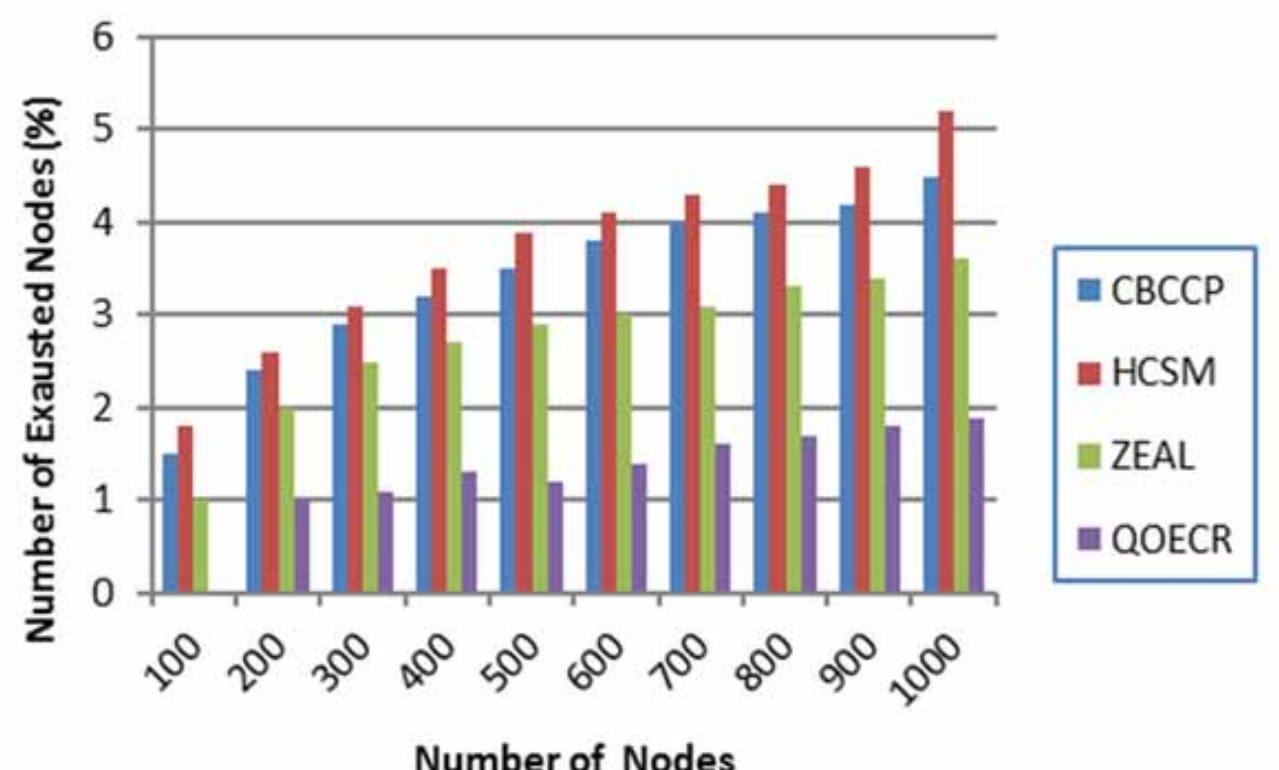




\section{CONCLUSION}

With an objective to improve the network lifetime and optimizing the energy consumption in IoT based WSN, QOECR is proposed and evaluated on diverse parameters in IoT based environment. QOECR includes three stages: pre-processing, setup and data aggregation stage. In pre-processing stage, QOECR outfits optimal route for sink to decrease power ingesting. In setup stage, QOECR recovers election of sub-sink nodes in tenacity to improve data delivery. QoS parameters including E2E delay, network lifetime, residual energy and throughput are assessed for enactment evaluation amid QOECR and CBCCP, HCSM and ZEAL. The outcomes exemplify that QOECR offers improved enactment in all facets. QOECR reduces E2E delay 30\%-35\%, enhances throughput 25\%-30\%, minimizes energy consumption 35\%-40\%, minimizes packet loss 28\%-32\%, improves PDR and prolongs network lifetime $32 \%-38 \%$. Results clearly verify that the proposed protocol successfully achieves its pre-determined objective of optimized energy consumption in IoT based WSN.

In the future, the proposed protocol will be applied on SNs with dissimilar energy necessities to assess its proficiency with other nature-inspired protocol. 


\section{REFERENCES}

Allam, A.H., Taha, M. \& Zayed, H.H. (2020). Enhanced Zone-Based Energy Aware Data Collection Protocol for WSNs (E-ZEAL). Journal of King Saud University-Computer and Information Sciences, 20(2), 1-11.

Amos, O. (2018). A System Dynamics Model for Mobile Industry Governance in the Context of the Kenyan Vision 2030. International Journal of System Dynamics Applications, 7(2), 81-100. doi:10.4018/IJSDA.2018040105

Anjum, S. S. (2019). Energy optimization of sustainable Internet of Things (IoT) systems using an energy harvesting medium access protocol. International Conference on Sustainable Energy and Green Technology, $268(2), 1-7$.

Anjum, S. S., Noor, R. M., \& Ahmedy, I. (2018). Energy optimization of sustainable Internet of Things (IoT) systems using an energy harvesting medium access protocol. Sustainable Energy and Green Technology, 268(1), $1-8$.

Bekalli, M. E., \& Balboul, Y. (2019). Energy harvesting based WBANs: EH optimization methods. Microwave Engineering. Communications Systems, 151(1), 1040-1045.

Bellavista, P., Berrocal, J., Corradi, A., Das, S. K., Foschini, L., \& Zanni, A. (2019). A survey on fog computing for the Internet of Things. Pervasive and Mobile Computing, 52(2), 71-99. doi:10.1016/j.pmcj.2018.12.007

Chithalru, P., Turjman, F. A., \& Kumar, M. (2020). I-AREOR: An Energy-balanced Clustering Protocol for implementing Green IoT in smart cities. Sustainable Cities and Society, 20(1), 1-30. doi:10.1016/j. scs.2020.102254

Chithaluru, P., Tiwari, R., \& Kumar, K. (2019). AREOR-Adaptive ranking-based energy efficient opportunistic routing scheme in Wireless Sensor Network. Computer Networks, 162(2), 1-13. doi:10.1016/j.comnet.2019.106863

Devi, M. D., Geetha, K., \& Saryanadevi, K. (2017). Content Based Routing Using Information Centric Network for IoT. Advances in Computing \& Communications, 115(1), 707-714. doi:10.1016/j.procs.2017.09.145

Farahani, B., Firouzi, F., Chang, V., Badaroglu, M., Constant, N., \& Mankodiya, K. (2018). Towards fog-driven IoT eHealth: Promises and challenges of IoT in medicine and healthcare. Future Generation Computer Systems, 78(2), 659-676. doi:10.1016/j.future.2017.04.036

Galli, B. J. (2019). Application of System Engineering to Project Management: How They Relate and Overlap. International Journal of System Dynamics Applications, 8(1), 79-93. doi:10.4018/IJSDA.2019010105

Galli, B. J. (2020). Continuous Technological Improvement Using Systems Engineering Principles to Achieve Sustainability: An Investigation into Related Literature. International Journal of System Dynamics Applications, 9(3), 1-25. doi:10.4018/IJSDA.2020070101

Haseeb, K., Din, I. U., \& Almogren, A. (2020). An Energy Efficeint and Secure IoT based WSN Framework: An Application to Smart Agriculture. IoT-Based Precision Agriculture, 20(7), 1-15.

Jain, B., Brar, G., Malhotra, J., \& Rani, S. (2017). Novel Approach for Smart Cities in Convergence to Wireless Sensor Networks. Sustainable Cities and Society, 17(2), 1-34. doi:10.1016/j.scs.2017.08.005

Kizito, A., \& Semwanga, A. R. (2020). Modeling the Complexity of Road Accidents Prevention: A System Dynamics Approach. International Journal of System Dynamics Applications, 9(2), 222-226. doi:10.4018/ IJSDA.2020040102

Kumar, A., \& Aanbanandam, R. (2019). Multimodal Freight Transportation Strategic Network Design for Sustainable Supply Chain: An OR Prospective Literature Review. International Journal of System Dynamics Applications, 8(2), 19-35. doi:10.4018/IJSDA.2019040102

Kumar, G., \& Rishiwal, V. (2019). Malicious User Detection by web Mining based Artificial Intelligence Technique. International Journal of Uncertainty, Fuzziness and Knowledge-based Systems, 134(1), 178-185.

Kumar, L., \& Rishiwal, V. (2019). Design of retrievable data perturbation approach and TPA for public cloud data security. Wireless Personal Communications, 108(1), 235-251. doi:10.1007/s11277-019-06399-7

Kumari, A., Tanwar, S., Tyagi, S., \& Kumar, N. (2018). Fog computing fir Healthcare 4.0 environment: Opportunities and challenges. Computers \& Electrical Engineering, 72(1), 1-13. doi:10.1016/j.compeleceng.2018.08.015 
Lin, Z., Li, G., \& Li, J. (2020). Cross-layer energy optimization in cooperative MISO wireless sensor networks. Computer Communications, 157(1), 351-360. doi:10.1016/j.comcom.2020.04.034

Manshahia, M. S. (2018). Grey Wolf Algorithm based Energy-Efficient Data Transmission in Internet of Things. Emerging Information. Communication and Networks, 160(2), 604-609.

Model and Simulate Dynamic System behavior with MATLAB. (n.d.). https://in.mathworks.com/discovery/ modeling-and-simulation.html

Moutaib, M., Fattah, M., \& Farhaoui, Y. (2020). Internet of Things:Energy Consumption and Data Storage. Big Data and Business Intelligence, 175, 609-614.

Mudasser, A.W., Rasool, S.M. \& Gafoor, A.A.A. (2019). An Energy Efficient Routing Protocol for WSN Assisting IoT. International Journal of Innovative Technology and Exploring Engineering, 8(7S2), 668-673.

Oma, R., Nakamura, S., Duolikun, D., Enokido, T., \& Takizawa, M. (2018). An energy-efficeint model for fog computing in the Internet of Things (IoT). Internet of Things, 1(2), 14-26. doi:10.1016/j.iot.2018.08.003

Panda, M. (2019). Software Defect Prediction Using Hybrid Distribution Base Balance Instance Selection and Radial Basis Function Classifier. International Journal of System Dynamics Applications, 8(3), 53-75. doi:10.4018/IJSDA.2019070103

Qawy, A. S. H. A., Mursi, A. M. S., \& Tadisetty, S. (2020). Classification of Energy Saving Techniques for IoTbased Heterogeneous Wireless Nodes. Computing and Network Communications, 171(1), 2590-2599.

Qureshi, K. N., Bashir, M. U., Lloret, J., \& Leon, A. (2020). Lioret, (2020). Optimized Cluster-Based Dynamic Energy-Aware Routing Protocol for Wireless Sensor Networks in Agriculture Precision. Journal of Sensors, 2020(1), 1-19. doi:10.1155/2020/9040395

Rani, S., Malhotra, J., \& Talwar, R. (2015). Energy efficient chain based cooperative routing protocol for WSN. Applied Soft Computing, 15(1), 1-12. doi:10.1016/j.asoc.2015.06.034

Rani, S., Talwar, R., \& Malhotra, J. (2020). A Novel Scheme for an Energy Efficient Internet of Things Based on Wireless Sensor Networks. Journal of Sensors, 15(1), 1-25. PMID:26569260

Sadek, R. A. (2018). Hybrid energy aware clustered protocol for IoT heterogeneous network. Future Computing and Informatics Journal, 3(2), 166-177. doi:10.1016/j.fcij.2018.02.003

Sharma, A., Bansal, A., \& Rishiwal, V. (2020). SBADR: Stable and bandwidth aware dynamic routing protocol for mobile ad hoc network. International Journal of Pervasive Computing and Communications, 81(3), 566-581.

Singh, O., \& Rishiwal, V. (2019). Scalable Energy Efficient Routing Mechanism Prolonging Network Lifetime in Wireless Sensor Networks. International Journal of Systems, Control and Communications, 2(2), 1-17.

Singh, O., \& Rishiwal, V. (2019). QoS Aware Multi-hop Multi-path Routing Approach in Wireless Sensor Networks. International Journal of Sensors, Wireless Communications and Control, 9(1), 43-52. doi:10.2174 /2210327908666180703143435

Singh, O., Rishiwal, V., \& Kumar, L. (2019). Secure Energy Aware Routing in Wireless Sensor Networks. Internet of Things: Smart Innovation and Usages, 1(1), 1-6.

Srinidhi, N.N., Kumar, S.M.D. \& Venugopal, K.R. (2019). Network optimizations in the Internet of Things: A review. Engineering Science and Technology, an International Journal, 22(1), 1-21.

Sudhari, R. P. M., \& Jaikumar, K. (2019). IoT assisted Hierarchical Computation Strategic Making (HCSM) and Dynamic Stochastic Optimization Technique (DSOT) for energy optimization in wireless sensor networks for smart city monitoring. Computer Communications, 19(1), 1-25.

Tekin, . (2020). IoT Activities Tuning for Energy Consumption. Artificial Intelligence \& Internet of Things, $175(1), 566-571$.

Viejo, A., \& Sanchez, D. (2019). Secure and privacy-preserving orchestration and delivery of fog-enabled IoT services. Ad Hoc Networks, 82(1), 113-125. doi:10.1016/j.adhoc.2018.08.002 
Yang, T., Xiangyang, X., Peng, L., Tonghui, L., \& Leina, P. (2018). A secure routing of wireless sensor networks based on trust evaluation model. Information and Communication Technology, 131(1), 1156-1163. doi:10.1016/j. procs.2018.04.289

Yassine, A., Singh, S., \& Hussain, M. S. (2018). IoT Big Data Analytics for Smart Homes with Fog and Cloud Computing. Future Generation Computer Systems, 91(1), 563-573.

Zhang, J. (2020). Real-time detection of energy consumption of IoT network nodes based on artificial intelligence. Computer Communications, 153(1), 188-195. doi:10.1016/j.comcom.2020.02.015

Vinay Rishiwal is working as Professor in Department of Computer Science and Information Technology, Faculty of Engineering and Technology, MJP Rohilkhand University, Bareilly. He has 20 years teaching and research experience. He received his Bachelor in Engineering (Computer Science and Engineering) in year 2000 from SRMS College of Engineering and Technology, Bareilly, India. He received his Ph.D. in 2011 from Gautam Buddh Technical University, Lucknow, India. He has more than 80 research publications in peer reviewed journal and conferences. He is reviewer/guest editor of many international journals. He has guided many students leading to M.E.MM.Tech and Ph.D. His research interests includes Quality of Service Issues in Advance Wireless Networks, Social networks and Security aspects in cloud computing. He is a senior member of IEEE. He has worked as the convener of IEEE UP Section for Web/NL and Student Activities Committee. He is general/conference chair of many IEEE Conferences.

Preeti Yadav is working as an Assistant Professor in the Department of CSIT, FET, MJP Rohilkhand University, Bareilly-243006, Uttar Pradesh, India. Currently, she is pursuing her PhD from Indian Institute of Technology Roorkee, Saharanpur Campus, Saharanpur, Uttar Pradesh, India. She has Obtained her B.Tech in CSE from SRMS CET, Bareilly in year 2007 and her M. Tech in CSE from Integral University in year. Her research interest is in Localization Problem in Wireless Sensor Network and Routing in Wireless Network.

Dr. Omkar Singh, Ph.D. is working as an Assistant Professor in the Department of Computer Science, Bareilly College, Bareilly, Uttar Pradesh, India. He has 08 years of teaching and research experience. He received his Bachelor of Science from MJP Rohilkhand University, Bareilly, India in the year 2007. He received Master of Computer Application with honor's from Gautam Buddh Technical University, Lucknow, India in the year 2011 and received his Ph.D. in Computer Science and Information Technology from MJP Rohilkhand University, Bareilly in the year 2021. He also qualified UGC-NET exam in the subject 'Computer Science and Applications' in the year 2012. He has published more than 35 research papers in various repute journals/conferences and 02 patent into her credit. He is reviewer/guest editor of many journals/conferences. His research interest includes Wireless Sensor Networks, Mobile Adhoc Networks, Vehicular Networks, Cloud Computing and Internet of Things.

B G Prasad is working as a Professor in Department of Computer Science and Engineering, B.M.S. College of Engineering, Bengaluru. He received B.E. degree in Computer Science \& Engineering during 1987 and M. Tech degree in Computer Science \& Engineering from IIT Delhi during 1992 and Ph.D. degree in Image Processing from IIT Delhi during 2003. His area of interests are Machine Learning, Image Processing, Computer Vision and Computer Networks. 